with the hazard of mortality (HR 2.23; 95\% CI 1.97 - 2.52). There was a progressive increase in mortality across the strata of hs-cTnI concentration (figure 1). Furthermore the $\log (10)$ hs-cTnI concentration was an independent predictor of the hazard of one year mortality (HR 1.77; 95\% CI 1.64 1.91). The discriminative ability of hs-cTnI for one year mortality was good with an AUC of 0.75 (95\%CI $0.73-0.76$ ). Further, the $\log (10)$ hs-cTnI concentration was independently associated with mortality across all three locations and most strongly in the OPD cohort (IPD HR 1.49; 95\% CI 1.33 1.67, OPD HR 2.44; 95\% CI 1.95 - 3.04, ED HR 1.99; $95 \%$ CI 1.76 - 2.25).

Conclusion In a large, unselected hospital population of both in- and out-patients, 18,282 (91.4\%) of whom there was no clinical indication for testing, hs-cTnI concentration was independently associated with one year mortality. These data suggest that hs-cTnI may have a role as a biomarker of future risk.

Conflict of Interest Beckman Coulter paid for all of the assays used in our studies but had no other role in the studies

\section{FEMALE SPEAKER REPRESENTATION AT NATIONAL CARDIOLOGY CONFERENCES}

${ }^{1}$ Rebecca Dobson, ${ }^{1}$ Clare Appleby, ${ }^{2}$ Rachel H Pathimagaraj. 'Liverpool Heart \& Chest Hospital, Liverpool, UK; ${ }^{2}$ University of Manchester

\subsection{6/heartjnl-2021-BCS. 182}

Introduction Women are under-represented in cardiology in the UK $(28 \%$ of cardiology trainees and $13 \%$ of cardiology consultants are female despite women accounting for $>50 \%$ of medical students). Lack of female role models is one of the commonly cited reasons for the lack of women within the field. Fair representation of female speakers at national cardiology conferences is therefore important to increase the visibility of women within cardiology. We assessed the extent of female representation at UK national cardiology conferences over the last 4 years.

Methods We requested the past programmes for annual conferences from 8 national cardiology societies in the UK. The programmes were reviewed and for each chair, panellist, operator and speaker, a binary definition of gender was assigned (female or male). In cases where gender was unclear from the name, the General Medical Council (GMC) register was referred to. For those not on the GMC register, we searched for the individual online. In any remaining cases of ambiguity, we selected the gender most commonly associated with that name. Speakers presenting abstracts were excluded.

Results Programmes were obtained from 7 societies (British Cardiovascular Society (BCS), British Cardiovascular Intervention Society (BCIS), British Heart Rhythm Society (BHRS), British and Irish Hypertension Society (BIHS), British Society of Cardiovascular Magnetic Resonance (BSCMR), British Society of Echocardiography (BSE), and British Society for Heart Failure (BSH). A total of 25 annual conferences were analysed. There were a total of 3959 slots; 982 of these were female (25\%). Of the 951 chair slots, 224 (24\%) were female. There were 3007 speaker/panellist slots, of which 760 (25\%) were female. See table 1 below for details.

Conclusion There is significant variation in the proportion of female speakers between societies (11-45\%). Specialties with the lowest numbers of female consultants had the lowest

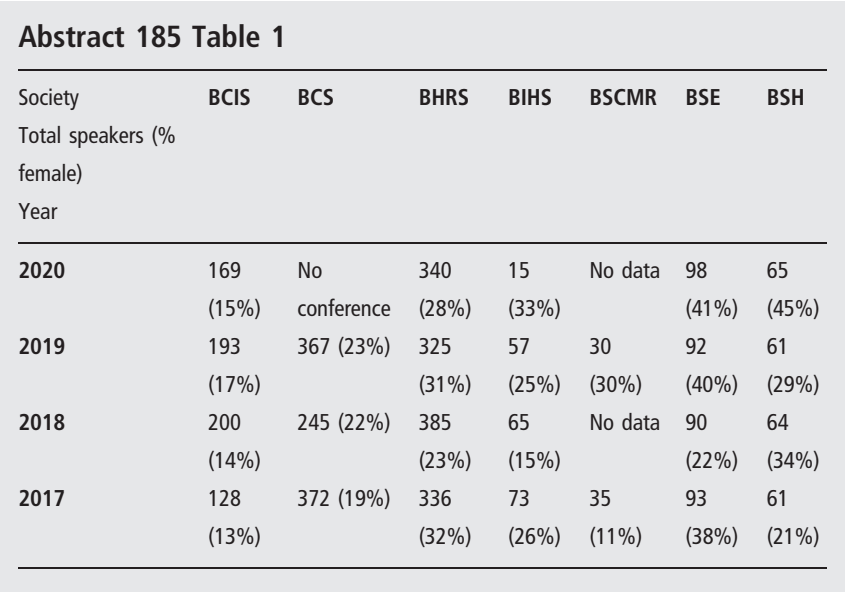

female representation, however BCS which represents all subspecialties also had poor female speaker representation. No clear improvement in female numbers occurred from 20172020. As part of a drive to facilitate gender equality within cardiology, conference committees need to ensure that speaker panels are gender balanced to enhance visibility of women within the specialty.

Conflict of Interest None

\section{EFFECTS OF AN ESTABLISHED DRUG ON SMAD SIGNALLING PATHWAY IN THE RAT MODEL OF MONOCROTALINE PULMONARY HYPERTENSION}

${ }^{1}$ Khaled Habas, ${ }^{2}$ Özlem Eklioğlu, ${ }^{1}$ Talat Nasim. ' University of Bradford, Bradford, UK; ${ }^{2}$ Anadolu University Faculty of Pharmacy Department of Pharmaceutical Toxicology Eskisehir/Turkey

\subsection{6/heartjnl-2021-BCS.183}

Introduction Pulmonary arterial hypertension (PAH) is a complex multifactorial disease with both genetic and environmental dynamics contributing to disease progression that characterized by unbalanced proliferation and apoptosis of pulmonary arterial smooth muscle cells (PASMCs) (Nasim et al., 2012). Mutations in the bone morphogenetic protein receptor type II (BMPRII) gene have been associated with development of familial pulmonary artery hypertension $(\mathrm{PAH})$. The function of SMAD signal transduction during the pulmonary vasculature and the role BMPRII mutations in the development of PAH are not fully understood. However, drug or toxin-induced $\mathrm{PAH}$ has been characterized by raised pulmonary arterial resistance leading to right heart failure. Aim and

Methods in this study, the monocrotaline (MCT) model of PAH was used to examine alterations in SMADs (SMAD3 and SMAD1/5) signal transduction pathways in vivo. The SMAD signalling pathways were investigated in lungs harvested from rats treated with a single $50-\mathrm{mg} / \mathrm{kg}$ of MCT and an established drug at concentrations of 0.5 and $1 \mathrm{mg} / \mathrm{Kg}$. The level of phosphorylation of SMAD3 and SMAD1/5 were detected by Western blot and the expression BMPR2, Id1, Pai1 transcripts was measured using quantitative real time PCR (qPCR).

Results MCT-treated rats decreased the level of SMAD 3 phosphorylation, which was restored following the treatment with the established drug. The drug also modulated the expression of SMAD target genes. 


\section{Correction: 185 Female speaker representation at national cardiology conferences}

Dobson R, Appleby C, Pathimagaraj R. 185 Female speaker representation at national cardiology conferences. Heart 2021;107:A144. doi:10.1136/heartjnl-2021-BCS.182

This Abstract has been corrected since it was first published. Author name 'Rachel Pathimagaraj' has been updated to 'Rachel H Pathimagaraj'.

(c) Author(s) (or their employer(s)) 2021. No commercial re-use. See rights and permissions. Published by BMJ.

Heart 2021;107:e9. doi:10.1136/heartjnl-2021-BCS.182corr1

A) Check for updates 